\title{
EVIDENCE OF A SOLVENT SCREEN EFFECT AFFECTING THE REDOX PROPERTIES OF CO(II) IONS IN COAPO-37, COAPO-40 AND COIST-2 (AEN), BY CYCLIC VOLTAMMETRY
}

\author{
Gabelica, Z. ${ }^{1 *}$, Louati, A. ${ }^{2}$, Borges, C. $^{3}$, Ribeiro, M.F. ${ }^{3}$, Lourenço, J.P. ${ }^{4}$ and Murphy, D.M. ${ }^{5}$ \\ ${ }^{1 *}$ Corresponding author:Université de Haute Alsace, ENSCMu, G.S.E.C., 3, Rue A. Werner, F-68093 Mulhouse Cedex, \\ France. Fax +33-3-8933-6815. E-mail: Z.Gabelica@uha.fr \\ ${ }^{2}$ ENSCMu, Lab. Electrochimie Analytique, 3, Rue Alfred Werner, F-68093 Mulhouse, France. \\ ${ }^{3}$ Instituto Superior Técnico, Dept Eng. Quimica, Av. R. Pais, P-1049-001, Lisboa, Portugal. \\ ${ }^{4}$ Universidade do Algarve, Dept Química e Bioquímica, P-8000-117 Faro, Portugal. \\ ${ }^{5}$ Cardiff University, Department of Chemistry, PO Box 912, Cardiff CF10 3TB, UK.
}

\begin{abstract}
Cyclic voltammetric experiments of framework Co(II) ions in three different CoAPO structures have provided evidence for a screening effect created by solvent or template molecules which results in a facile oxidation (and a difficult reduction) of the Co(II) ions incorporated into the most open (the less strained) structures. An apparent contradiction of these findings with respect to theoretical expectations is discussed.
\end{abstract}

\section{INTRODUCTION}

During a systematic exploration of non conventional routes to generate hydrothermally aluminophosphate type materials using both short chain amines and water as co-templates, we succeeded in the preparation of a series of new aluminophosphate phases [1]. One such phase, called IST-1, appeared to be novel [2], while another, called IST-2, produced an XRD pattern closely related to that of CFSAPO-1 [3] that probably involves an AEN type topology, along with a series of other isostructural compounds, including AlPO-EN3 [4], MSC-1 [5], GaPO $-\mathrm{M} 2$ [6], JDF-2 [7], AlPO-53(A) [8] or UiO-12 [9]. The thorough characterization of this compound [10] allowed us to subsequently conclude that its framework type is also closely related to that of AlPO-53 (A). The air-calcined IST-2, hereafter labelled IST-2(B), exhibited a new structure that is actually analogous to that of AlPO-53(B) (the calcined AlPO-53(A) [11]), thereby confirming its AEN topology.

IST-2 proved to be a convenient host for the accommodation and stabilization of various transition metal ions, such as $\mathrm{Cu}(\mathrm{II})$ [12] or $\mathrm{Co}(\mathrm{II})$ [13], yielding respectively the corresponding pure and highly crystalline MeAPO's without any metal-bearing side phase. To the best of our knowledge, there is no other example reporting the incorporation of a divalent metal ion into the framework of an AEN type material.

Although the insertion of cobalt ions into the framework of several aluminophosphate materials has been well addressed [14-21], their coordination and redox properties are still controversial and seem to be dependent, in particular, on the structure itself [15,18-22].

During a preceeding study [13], we have evaluated by FTIR using gaseous NO as probe, the redox capability of $\mathrm{Co}$ (II) ions sited in the framework of partly air-calcined and also on $\mathrm{H}_{2}$-reduced CoIST-2, CoAPO-37 and CoAPO-40, and contributing to the Brønsted acidity of these materials (P-(OH)-Co(II) species). The consequent formation of dinitrosyl type species that could be quantified by FTIR [13] suggested that the framework $\mathrm{Co}(\mathrm{II})$ ions associated with hydroxyls bridging them and the neighboring $\mathrm{P}$ atoms (Brønsted acid sites) in CoAPO-37 and CoAPO-40 was more resistant to oxidation than in CoIST-2. Such observations were in line with earlier findings or predictions $[15,19]$ stating that the constraints existing in more dense $\mathrm{AlPO}_{4}$ structures lead to local distortions that can be responsible for a less steady stabilization of framework $\mathrm{Co}$ (II) ions, thus leading to their facile oxidation. In other words, the degree of distortion of the cobalt site and, hence, its ease to undergo oxidation, are dependent on the framework type and, more specifically, on the structure density [19]. 
In contrast to these findings, upon running the electrochemical oxidation of these $\mathrm{P}-(\mathrm{OH})-\mathrm{Co}(\mathrm{II})$ species remaining in the partly air-calcined CoIST-2, the anodic peak corresponding to $\mathrm{Co}(\mathrm{II}) \rightarrow \mathrm{Co}$ (III) oxidation occurred at a relatively high potential $(+1.18$ Volt $)$, suggesting that we should deal with a significantly more open structure than that of AlPO-53(B) [8].

To confirm the real influence of the $\mathrm{AlPO}_{4}$ structure on the overall stability of framework P-(OH)-Co(II) species and to explain the apparently contradictory conclusions drawn from FTIR and our preliminary electrochemical measurements [13], we report here a more in depth cyclic voltammetric evaluation on the ease of oxidation and/or reduction of framework $\mathrm{Co}$ (II) ions incorporated into the as-synthesized and partly air-calcined CoIST-2, CoAPO-37 and CoAPO-40 compounds.

\section{EXPERIMENTAL}

\section{Samples}

CoIST-2 was prepared hydrothermally from gels involving, along with $\mathrm{Al}, \mathrm{P}$ and $\mathrm{Co}(\mathrm{II})$ sources, methylamine (main template), water (solvent and co-template) and TEAOH (co-additive), as described elsewhere [13]. The pore structure of the calcined CoIST-2, analogous to AlPO-53(B), consists of two 8-ring channels intersecting to generate a 2D channel system with pore openings of $4.3 \times 3.1 \AA$ and $2.7 \times 5.0 \AA$ $[8,11]$. It has a framework density (FD) of 19.7 and the unit cell composition $\left(\mathrm{Co}_{0.02} \mathrm{Al}_{0.48} \mathrm{P}_{0.50}\right) \mathrm{O}_{2}[13]$.

CoAPO-37 (FAU) and CoAPO-40 (AFR), were prepared following the original procedures described previously [16,22]. Their unit cell composition was respectively $\left(\mathrm{Co}_{0.02} \mathrm{Al}_{0.48} \mathrm{P}_{0.50}\right) \mathrm{O}_{2}$, [22] and $\left(\mathrm{Co}_{0.01} \mathrm{Al}_{0.49} \mathrm{P}_{0.50}\right) \mathrm{O}_{2}[16]$. Table 1 compares the structural characteristics of the three compounds.

Table 1.

\begin{tabular}{|c|l|c|}
\hline Topology & \multicolumn{1}{|c|}{ Channel system $^{\mathrm{a}}$ ) } & Framework density \\
\hline AEN B $^{\mathrm{b}}($ CoIST-2) & $(100) \underline{\mathbf{8}} 3.1 \times 4.3^{*}----(010) \underline{\mathbf{8}} 2.7 \times 5.0^{*}$ & 19.7 \\
\hline AFR (CoAPO-40) & $(001) \underline{\mathbf{1 2}} 6.7 \times 6 . *^{*---}(010) \underline{\mathbf{8}} 3.7 \times 3.7^{*}$ & 15.0 \\
\hline FAU (CoAPO-37) & $<111>\mathbf{1 2} 7.4 \times 7.4^{* *}$ & 12.7 \\
\hline
\end{tabular}

(a) International abbreviations as in [11]

(b) Structure data of AlPO-53 (B), which corresponds to our air-calcined CoIST-2

The redox ability of both the as synthesized and (partly) air-calcined CoIST-2, along with the above mentioned two other CoAPO samples taken as comparative references, was confirmed by cyclic voltammetry involving modified carbon paste electrodes, using the procedure described below. Samples were only partly calcined so that the $\mathrm{P}-(\mathrm{OH})-\mathrm{Co}(\mathrm{II})$ species are still present in the template-free structures along with the P-O-Co(III) entities generated upon dehydration and cobalt oxidation [20]. In such a case, the redox ability of framework $\mathrm{P}-(\mathrm{OH})-\mathrm{Co}(\mathrm{II})$ species could also be probed in the calcined samples where the influence of neighboring molecules (water in place of templates) could be different .

\section{Cyclic voltamperommetric measurements}

Voltammetric experiments were performed on an EG\&G 263 A potentiostat modulated by EG\&G PAR M270 software. All measurements were carried out at ambient temperature with a conventional three electrode configuration, consisting of a platinum wire as auxiliary electrode, modified sample-paste carbon as working electrode, and a saturated calomel reference electrode (SCE). Pure water (18 M $\Omega \mathrm{cm}$, Millipore Milli-Qplus) was used throughout. Potassium chloride (Fluka, Puriss p.a.) was used as supporting electrode. The working electrode was set up by using the following home made procedure: the body of the carbon paste electrode, consisting of a Teflon rod (diameter $1 \mathrm{~cm}$ ) into which the carbon paste was filled and of another 3 $\mathrm{mm}$ diameter glassy carbon rod, was inserted into the other end of the electrode so as to make the electrical contact. The modified sample-paraffin oil-carbon powder paste was prepared by thoroughly admixing in a mortar the desired amount of sample (typically $20-25 \mathrm{mg}$ ) with respectively $60-55 \mathrm{mg}$ of carbon. $35-40 \mu \mathrm{lof}$ paraffin oil were then added until a homogeneous paste was obtained. The paste was packed into the electrode and a fresh surface was rapidly generated through rolling out a small plug of the paste with the glassy carbon rod. The plug was subsequently scrapped off and smoothed with the help of a piece of white paper so as to obtain a homogeneous and uniform electrode surface. 


\section{RESULTS AND DISCUSSION}

Theoretical expectations for the stability of $\mathrm{P}-(\mathrm{OH})-\mathrm{Co}(\mathrm{II})$ species in different pore structures

Our previous comparison on the behaviour of CoIST-2, CoAPO-37 and CoAPO-40 upon NO adsorption [13] suggested that the framework $\mathrm{Co}(\mathrm{II})$ ions in CoAPO-37 and CoAPO-40 were more resistant to oxidation than in CoIST-2. This was inferred from FTIR experiments, where a much higher decrease of the bands assigned to dinitrosyls grafted onto the $\mathrm{P}-(\mathrm{OH})-\mathrm{Co}(\mathrm{II})$ entities remaining in CoIST-2 was observed when the NO pressure was increased, compared to the other structures [13]. A similar behaviour was already reported for CoAPO-18 (framework density of 14.8) when compared with CoAPO-5 (framework density of 17.3) $[20,21]$.

The resistance of framework $\mathrm{Co}(\mathrm{II})$ ions to oxidation seems to be highly dependent on the porous structure, as reported by Verberckmoes and coworkers [19] and Barrett et al. [15] and is probably related to the constraints that exist in dense structures. A strained framework would indeed involve local distortions that would more readily destabilize the lattice $\mathrm{Co}(\mathrm{II})$ ions and thereby lead to their easier oxidation under appropriate conditions (e.g. P-(OH)-Co(II) $\rightarrow$ P-O-Co(III) and the consequent loss of the Brønsted acidity). Similarly, the degree of oxidation of $\mathrm{Co}$ (II) to $\mathrm{Co}$ (III) (for a given heat treatment in air) should follow the same sequence [15]. All these trends are expected at the gas-solid interface, in the absence of any coordinated solvent molecule.

\section{Comparative electrochemical behavior of CoAPO-37, CoAPO-40 and CoIST-2}

Figure 1 shows the cyclic voltammograms of the as synthesized CoAPO-37, CoAPO-40 and CoIST-2 embedded in a carbon paste modified electrode in the $0-1.5$ Volt range (anodic range).
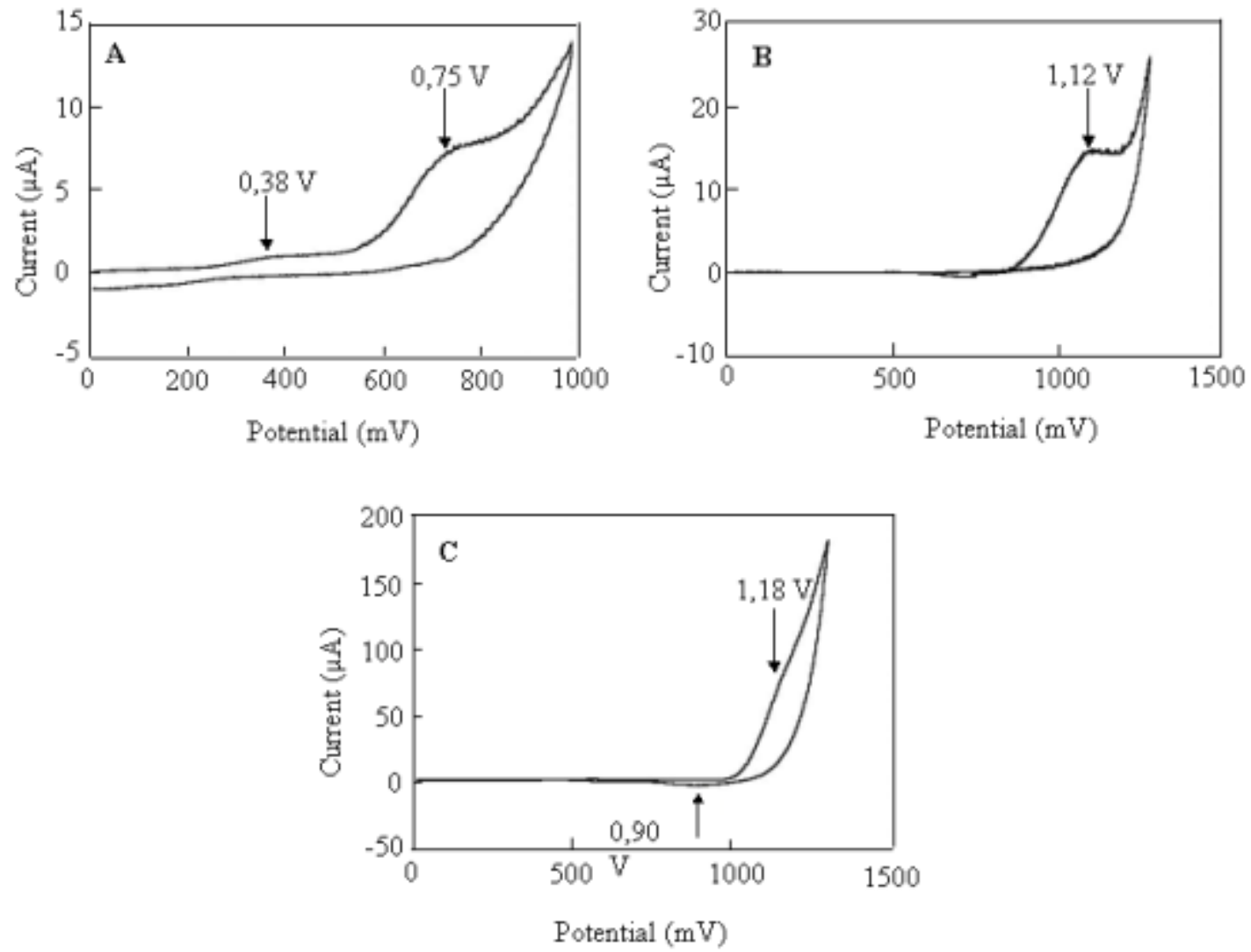

Figure 1. Cyclic voltammogram (anodic oxidation followed by cathodic reduction) of as-synthesized (A): CoAPO-37; (B): CoAPO-40 and (C): CoIST-2, recorded with a modified carbon paste electrode in $0.1 \mathrm{M} \mathrm{KCl} \mathrm{electrolyte,} \mathrm{using} \mathrm{a}$ $25 \mathrm{mV} \mathrm{s}^{-1}$ scan rate.

The respective oxidation peaks of the three compounds (showing a decreased opening of the pore structure in that order, as reflected by the increase of their framework density (Table 1)), was observed at $0.75,1.12$ and $1.18 \mathrm{~V}$ respectively. Another weak oxidation at $0.38 \mathrm{~V}$ is also seen in the case of CoAPO-37. 
While CoAPO-37 collapsed upon air oxidation and further exposure to atmospheric moisture, calcined CoAPO-40 and CoIST-2 show a $\mathrm{Co}(\mathrm{II}) \rightarrow \mathrm{Co}(\mathrm{III})$ oxidation at 0.90 and 1.10 Volt respectively (figure not shown), thus confirming the sequence found for the as-synthesized materials and confirming that the air treatment did not completely oxidize all framework Co(II). It should also be borne in mind that although the $\mathrm{P}-(\mathrm{OH})-\mathrm{Co}(\mathrm{II})$ species are still partly present in the calcined samples (Brønsted acidity was confirmed in each case by FTIR [13]), the electrochemically generated Co(III) species are not necessarily the same as those generated by the heat treatment (namely framework P-O-Co(III)), but can possibly result in an irreversible cobalt extraction from the framework upon anodic oxidation.

The above findings first suggest that the most open structure (ie., the less strained structure) undergoes the easiest oxidation. Such conclusions apparently contradict our FTIR data for NO sorption and also the conclusions by Verberckmoes et al. [19], who state that the sequence of oxidative ability generally follows the decrease of pore size or the increase of the structure density. In other words, one should have expected to observe the reverse trend, namely an easier oxidation in a more dense structure (CoIST-2) and its better stabilization in the two other more open structures.

To reconcile both sets of data, one must consider that in the electrochemical experiments, the cobalt species are surrounded (solvated) by a coordination sphere of solvent molecules, either consisting of template entities in the as-synthesized phases or by water molecules from the electrolytic solution in the calcined samples. Such molecules can generate a partial screening effect around the ion, which alters the chemical potential of the core ion due to the polarizability of the solvent, and thereby results in a less efficient removal of the electron during oxidation, unlike the situation encountered in the absence of any solvent or screening molecules. This screening effect by the solvent molecules should only be effective in the electrochemical experiments, and will be expected to vary depending on the structure as the ion becomes more readily solvated in the open structure (and therefore more easily oxidised) compared to the less readily solvated ion in the dense structure (ie., more difficult to oxidise), since the solvent molecules are acting as the electrolytic solution. In other words, the more solvent molecule coordinated to the ion, the more efficient the electrochemistry. In the FTIR experiment, NO is probing the same type of (remaining) $\mathrm{Co}(\mathrm{II})$ hydroxyl-bridged sites but these latter sites are not influenced, perturbed or screened by any solvent or template molecule in the vacuum-treated compound before NO sorption. Another way to explain this is to consider that the chemical potential of the cobalt ions at the liquid-solid interface in the electrochemical experiment should be probably different compared to the gas-solid interface experienced in the NO experiment, and this likely manifests itself in the different oxidative stability of $\mathrm{Co}$ (II) ions.

The fact that the observed trend follows the structure density and is not random for the three compounds, indicates that the pore structure should indeed play some major role in controlling the extent of this solvent screening effect. This also infers that the same kind of $\mathrm{P}-(\mathrm{OH})-\mathrm{Co}(\mathrm{II})$ species are stabilized in each framework prior to their probing (FTIR) or oxidation (voltammetry), independently of the screening effect. Indeed, while the stabilization of (part of) Co(II) by oxygen vacancies [15] in the dehydroxylated calcined phases and yielding Lewis-type sites [20] was also detected in the NO-FTIR experiments [13], these dinitrosyl species are not taken into account in the present comparison.

The subsequent reduction of Co(III) species is not seen on the cathodic part of the cyclic voltammograms for the two more open structures, namely CoAPO-37 (Fig. 1a) and CoAPO-40 (Fig.1b), confirming the irreversible oxidation of the corresponding $\mathrm{Co}$ (II) species, at least under our electrochemical conditions. In contrast, for CoIST-2, the small reduction peak (arrow in Fig 1c) can be related to the reversible reduction of a part of the $\mathrm{Co}(\mathrm{III})$ ions, possibly corresponding to the framework $\mathrm{P}-\mathrm{O}-\mathrm{Co}(\mathrm{III}) \rightarrow \mathrm{P}-(\mathrm{OH})-\mathrm{Co}(\mathrm{II})$ transition that can be (partly) reversible in the voltammetric experiments.

Although the solvent screening effect does not totally provide an explicit mechanism for a less favourable stabilization of $\mathrm{Co}$ (II) in a more open structure, a more difficult oxidation should, from an electrochemical perspective, correspond to an easier reduction. This is exactly what was observed during the separate cathodic reduction followed by the cyclic re-oxidation of the three as-synthesized phases (Fig 2).

While two reduction peaks are detected for CoAPO-37 (Fig 2a), only one single reduction is seen in the case of CoAPO-40 and CoIST-2 (at respectively -1.15 V, Fig. $2 b$ and at -0.99 V, Fig. $2 c$ ). Such a reduction corresponds here to $\mathrm{Co}(\mathrm{II}) \rightarrow$ metallic $\mathrm{Co}$. We have also confirmed that, in the case of CoAPO-37, the two peaks observed at -0.83 and $-1.07 \mathrm{~V}$ can also be attributed to that type of reduction and not to a sequential and successive $\mathrm{Co}(\mathrm{II})$ to $\mathrm{Co}(\mathrm{I})$ and then to $\mathrm{Co}^{\circ}$ reduction [10]. This suggests that two structurally different cobalt species are present in this compound (see below).

Although such a reduction of Co(II) species to metallic cobalt could never be achieved in the high temperature conditions used in the case of other calcinations-reduction treatments achieved prior to the 
UV-Vis or FTIR investigations of the same samples [13], the trend observed during electrochemical experiments again corresponds to an easier reduction (and, hence, an easier framework extraction) of Co(II) when the structure is less open (CoIST-2) than for a more open and probably less strained structure (CoAPO-40). This backs up the conclusions drawn from the oxidation experiments performed on the same (screened) compounds.
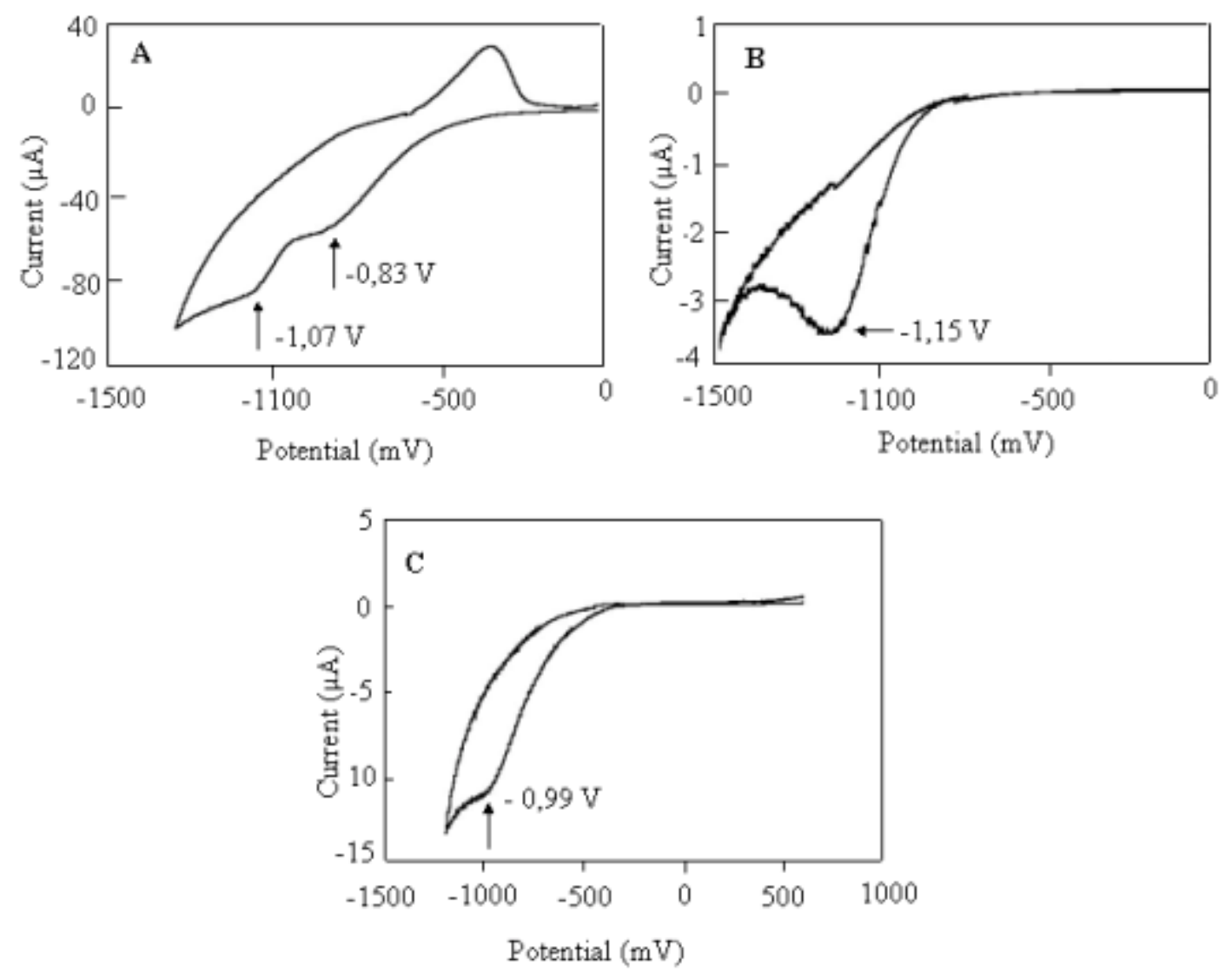

Figure 2. Cyclic voltammogram (cathodic reduction followed by anodic oxidation) of as-synthesized (A): CoAPO-37; (B): CoAPO-40 and (C): CoIST-2, recorded with a modified carbon paste electrode in $0.1 \mathrm{M} \mathrm{KCl} \mathrm{electrolyte,} \mathrm{using} \mathrm{a}$ $25 \mathrm{mV} \mathrm{s}^{-1}$ scan rate.

So far, CoAPO-37 does not seem to follow that trend. Indeed, it is mainly composed of (two types of) cages rather than really intersecting channels, which probably corresponds to a very different strain picture. The two differently structure-positioned P-(OH)-Co(II) species in the FAU topology (that respectively reduce at -0.83 and $-1.07 \mathrm{~V}$, Fig $2 \mathrm{a}$ ), possibly reflect their respective location in both the supercages and the sodalite cages, in that order. Two FTIR bands in the $2000-2300 \mathrm{~cm}^{-1}$ region attributed to two different $\mathrm{NO}^{+}$ species were also detected in the case of CoAPO-37 [10,13].

Further work is in progress to confirm the structural effects of cage-bearing porous materials.

\section{CONCLUSIONS}

The cyclic voltammetric evaluations on the ease of the $\mathrm{P}-(\mathrm{OH})-\mathrm{Co}(\mathrm{II})$ species, present in the framework of both the as-synthesized and calcined CoAPO-37, CoAPO-40 and CoIST-2 materials, to undergo oxidation (to $\mathrm{Co}(\mathrm{III})$ ) or reduction (to metallic $\mathrm{Co}$ ), suggest that the most open structure (the less strained) undergoes the easiest oxidation and thus the most difficult reduction. This conclusion apparently contradicts our previous experimental findings (FTIR- NO sorption data) and the theoretical predictions. To reconcile both sets of data, we have proposed that a coordination sphere of solvent molecules (water from the electrolytic solution for the calcined phases) or of template molecules (water, methylamine or tetraalkylammonium ions in the as-synthesized compounds) surrounding the cobalt species can generate some screening effect responsible for a less efficient removal (supply) of the electron during oxidation (reduction). The chemical potentials of the cobalt ions at the liquid-solid interface in the electrochemical experiments are probably 
different compared to the gas-solid interface experienced in the FTIR experiments, and this likely influences differently the stability of $\mathrm{Co}$ (II) ions towards oxidation or reduction. The fact that the trend follows the structure density indicates that the pore structure must play some role whereby the extent of screening is subtlety changed depending on the structure. This does not exclude the influence of other factors in controlling the stability of the ion, such as a different stabilization of Co(II) in the calcined phases by the framework atoms or even the presence of oxygen vacancies, which could ultimately influence, to some extent, the potential values in the voltammetric experiments.

Nevertheless, it can be proposed that voltammetry could prove a rapid, reliable and useful technique for the evaluation of the redox behaviour of other host heteroatoms and, more specifically, of transition metal ions sited in other porous (zeolite and aluminophosphate) frameworks and therefore should be of considerable potential interest in predicting various catalytic properties of such species.

\section{ACKNOWLEDGMENTS}

The Portuguese authors thank FCT, POCTI and FEDER for financial support.

\section{REFERENCES}

1. C. Borges, M.F. Ribeiro, C. Henriques, M.T. Duarte, J.P. Lourenço, Z. Gabelica, in preparation.

2. J.L. Jorda Moret, L.B. McCusker, C.Baerlocher, C.M. Morais, J. Rocha, C. Fernandez, C. Borges, J.P. Lourenço, M.F. Ribeiro, Z. Gabelica, submitted

3. J.B. Parise, Stud. Surf. Sci. Catal. 24 (1985) 271.

4. H. He, Y. Long, J. Inclusion Phenomena 5 (1987) 591.

5. A. Simmen, Ph.D Thesis, ETH, Zurich, Switzerland (1992)

6. F.P Glasser, R.A. Howie, Q.B. Kan, Acta Crystallogr., C50 (1994) 848.

7. A.M. Chippindale, A.V. Powell, R.H. Jones, J.M. Thomas, A.K. Cheetham, Q.S. Huo, R.R. Xu, Acta Crystallogr., C50 (1994) 1537.

8. R.M. Kirchner, R.W. Grosse-Kunstleve, J.J. Pluth, S.T. Wilson, R.W. Broach, J.V. Smith, Microporous Mesoporous Mater. 39 (2000) 319.

9. Q.O. Kongshaug, H. Fjellvag, B. Klewe, K.P. Lillerud, Microporous Mesoporous Mater. 39 (2000) 333.

10. C. Borges, Ph.D Thesis, Lisbon, Portugal, July 2003

11. Ch. Baerlocher, W.M. Meier, D. H. Olson, Atlas of Zeolite Structure Types, Elsevier, Amsterdam, 2001.

12. C. Borges, M.F. Ribeiro, C. Henriques, J.P. Lourenço, S. Valange, A. Louati, Z. Gabelica, D.M. Murphy, in preparation

13. C. Borges, M.F. Ribeiro, C. Henriques, J.P. Lourenço, A. Louati, D.M. Murphy, Z. Gabelica, submitted

14. V. Kurshev, L. Kevan, D.J. Parillo, C. Pereira, G.T. Kokotailo, R.J. Gorte, J. Phys. Chem. 98 (1994) 10160.

15. P.A. Barret, G. Sankar, C.R.A. Catlow, J.M. Thomas, J. Phys. Chem. 100 (1996) 8977.

16. J.P. Lourenço, M.F. Ribeiro, F.R. Ribeiro, J. Rocha, B. Onida, E. Garrone, Z. Gabelica, Zeolites 18 (1997) 398.

17. M. Hochtl, A. Jentys, H. Vinek, Microporous Mesoporous Mater. 31 (1999) 271.

18. J. Sponer, J. Cejka, J. Dedecek, B. Wichterlová, Microporous Mesoporous Mater. 37 (2000) 117.

19. A.A. Verberckmoes, B.M. Weckhuysen, R.A. Schoonheydt, Microporous Mesoporous Mater. 22 (1998) 165.

20. L. Marchese, J. Chen, J.M. Thomas, S. Collucia, A. Zecchina, J. Phys. Chem. 98 (1994) 13350.

21. E. Gianotti, L. Marchese, G. Martra, S. Coluccia, Catalysis Today 54 (1999) 547.

22. C.S. Costa, J.P. Lourenço, C. Henriques, A.P. Antunes, F.R. Ribeiro, M.F. Ribeiro, Z. Gabelica, Proc. 12th IZC, M.M.J. Treacy et al (Eds), MRS, Warrendale, 1999, p. 1771. 